\title{
UNA NUEVA INSTITUCIONALIDAD ACADÉMICA PARA LA GESTIÓN DE LA RESPONSABILIDAD SOCIAL DE LA UNIVERSIDAD.
}

R. R. LÓPEZ
Universidad de Córdoba (UCO)
ramon.rueda@uco.es
Artigo submetido em 13/08/2018 e aceito em 08/03/2019
DOI: $10.15628 /$ holos.2019.7624

\section{RESUMO}

La responsabilidad social de la Universidad es un paradigma de comportamiento universitario llamado a dar respuesta a las expectativas que la sociedad tiene depositadas en la Universidad. Sin embargo, con el propósito de profundizar los principios de libertad y autonomía universitaria y de democracia universitaria, capaces de dar sentido a un compromiso social de la Universidades, especialmente las públicas, es necesario, introducir en al ámbito de la propia gestión de la
Universidad vectores de comportamiento como la igualdad de género, el respeto a la diversidad y la pluralidad de cosmovisiones y el diálogo con los grupos sociales y de interés de la universidad que, junto a la garantía de un financiamiento público, hagan de la Universidad y de su responsabilidad social, una institución desde la cual abordar y dar respuesta a los retos presentes y futuros a los que la humanidad se enfrenta.

PALABRAS CLAVE: Responsabilidad social de la Universidad, igualdad de género, financiación pública, grupos de interés, pluralidad.

\section{A NEW ACADEMIC INSTITUTION FOR THE MANAGEMENT OF THE SOCIAL RESPONSIBILITY OF THE UNIVERSITY.}

\begin{abstract}
The social responsibility of the University is a paradigm of university behavior called to respond to the expectations that society has deposited in the University. However, with the purpose of deepening the principles of university freedom and autonomy and of university democracy, capable of giving meaning to a social commitment of the Universities, especially public ones, it is necessary to introduce into the scope of the University's own
\end{abstract}

management behavioral vectors such as gender equality, respect for diversity and plurality of worldviews and dialogue with social and interest groups of the university that, together with the guarantee of public funding, make the University and its social responsibility, an institution from which to approach and respond to the present and future challenges that humanity faces.

KEYWORDS: Social responsibility of the University, gender equality, public financing, stakeholders, plurality. 


\section{INTRODUCCIÓN}

Más allá de su interpretación como "servicio público», la educación superior es un "bien público» del que todas las partes involucradas son responsables. Este es el derecho irrenunciable del que se parte.

En su caso, las universidades, son las encargadas de realizar el servicio público de la educación superior. Especialmente las universidades públicas.

No existe, por tanto, un vínculo más poderoso entre las universidades y toda la sociedad que éste. Instancia desde la cual las universidades deberían asumir la responsabilidad como el principio regulador de su conducta.

Es desde estas reflexiones previas desde donde surge una nueva dimensión de la «autonomía con responsabilidad». Desde esta dimensión, más que gestionar sus impactos negativos, como se propone desde el modelo clásico de la RSU, la Universidad debe tomar consciencia de su papel en la sociedad interconectada, de tal manera que asuma un comportamiento proactivo con la intención de prevenir y, en último termino, ofrecer soluciones a las injusticias sociales, mediante sus funciones sustantivas (Tünnermann Bernheim, 2008a, 2008b).

Alcanzar este estado de "autonomía con responsabilidad» sólo es posible desde la libertad y desde la democracia institucional, principios insoslayables que deben concretarse, en el ámbito de la gestión institucional de la Universidad, a través de cuatro vectores fundamentales. En primera instancia la financiación de la universidad pública, en segunda instancia, y como ámbitos invocados a profundizar la democracia universitaria, la igualdad de género, el respeto a la diversidad y la pluralidad de cosmovisiones y el diálogo con los grupos sociales y de interés de la universidad.

Esta investigación tiene como objetivo, en el ámbito concreto de la gestión académica, proponer, a partir de una epistemología, ética y normativa, diferente a la de la Responsabilidad Social Corporativa, una nueva dimensión de la idea de universidad comprometida, o si así se prefiere, de Responsabilidad Social de la Universidad. Se trata, en definitiva, de ofrecer respuestas, desde un sentido práctico, a la noción de «autonomía con responsabilidad».

Para poder alcanzar este propósito resulta conveniente tomar en consideración la primera (1998) y la segunda (2009) Conferencia Mundial sobre la Educación Superior. En ambas es posible identificar un modelo de educación superior y de Universidad con un fundamento de carácter ético y humanista. Es, por tanto, en estos foros de discusión y reflexión, a los que, a pesar de la distancia temporal, es conveniente prestar atención para comenzar a hallar respuestas al propósito fijado.

Dicho esto, es preciso resaltar una diferencia esencial en cuanto a la primera y la segunda CMES. Así, si en el caso de esta primera Conferencia el formato elegido para trasladar sus resultados a los Gobiernos, los organismos internacionales, la comunidad educativa y la sociedad en general, fue el de una declaración, en el caso de la segunda fue, tan sólo, mediante un comunicado. Este factor, sin duda, debilita aún más la capacidad que tiene la CMES para generar compromisos entre los Estados firmantes. 
Este hecho es un indicador de los tensos debates que en el seno de aquella reunión se produjeron y, por extensión, de la dificultad para alcanzar un acuerdo. Especialmente, como así relata Carlos Tünnermann, entre aquellas posiciones lideradas por las delegaciones latinoamericanas que pedían (como así quedó recogido finalmente) que la educación superior fuera definida como "bien público», y las posturas de las delegaciones de países del marco de la OCDE que optaban por denominarla como "servicio público». Calificación ésta última que, en última instancia, hubiera permitido incluir, definitivamente a la educación superior en el Acuerdo General sobre el Comercio de Servicios de la OMC (Tünnermann Bernheim, 2010, p. 44).

Sin embargo, junto a este "activo», la falta de descripción y ambición del Comunicado del 2009 en comparación con la Declaración de 1998 en cuanto a las políticas de género en la educación superior, constituye uno de los principales «pasivos» del mismo (Sanchis Vidal, 2014, pp. 646-647).

En general, la segunda CMES, usando las palabras de Marco-Antonio Rodrigues Dias, no alcanzó "ni los objetivos ni la representatividad de la primera Conferencia” (Rodrigues Dias, 2010), algo con lo que coincide Tünnermann al señalar que el Comunicado de 2009 no tuvo "la riqueza conceptual y propositiva de la Declaración de 1998, que se constituyó en la brújula orientadora de los procesos de transformación de la educación superior en el mundo" (Tünnermann Bernheim, 2010, p. 44).

Sin embargo, el hecho de que autores como el propio Tünnermann o López Segrerara otorguen a la CMES un alto valor en cuanto al diseño de políticas y propuestas para la educación superior a nivel mundial, hacen de estas reuniones, especialmente de la primera, un referente alternativo en el que buscar respuestas a las preguntas formuladas anteriormente.

En este sentido, además de la protección de la educación superior a través de su definición como «bien público», el Comunicado de 2009 presentó la responsabilidad social de la educación superior como concepto que transciende al de pertinencia social de la universidad. Este hito es particularmente relevante en tanto en cuanto, la UNESCO trató de fijar la responsabilidad social de la educación superior a partir del criterio de pertinencia social definida en la Declaración de 1998, en unos años en los que, como se ha puesto de manifiesto en el epígrafe anterior, la Responsabilidad Social Universitaria asumía un carácter determinado.

De esta manera, el Comunicado inmediatamente después de su preámbulo señaló la responsabilidad social de la educación superior de la siguiente forma:

"1. La educación superior, en tanto que bien público, es responsabilidad de todas las partes interesadas, en particular de los gobiernos.

2. Ante la complejidad de los desafíos mundiales, presentes y futuros, la educación superior tiene la responsabilidad social de hacer avanzar nuestra comprensión de problemas polifacéticos con dimensiones sociales, económicas, científicas y culturales, así como nuestra capacidad de hacerles frente. La educación superior debería asumir el liderazgo social en materia de creación de conocimientos de alcance mundial para abordar retos mundiales, entre los que figuran la seguridad alimentaria, el cambio climático, la gestión del agua, el diálogo intercultural, las energías renovables y la salud pública. 
3. Los centros de educación superior, en el desempeño de sus funciones primordiales (investigación, enseñanza y servicio a la comunidad) en un contexto de autonomía institucional y libertad académica, deberían centrarse aún más en los aspectos interdisciplinarios y promover el pensamiento crítico y la ciudadanía activa, contribuyendo así al desarrollo sostenible, la paz y el bienestar, así como a hacer realidad los derechos humanos, entre ellos la igualdad entre los sexos.

4. La educación superior debe no sólo proporcionar competencias sólidas para el mundo de hoy y de mañana, sino contribuir además a la formación de una ciudadanía dotada de principios éticos, comprometidos con la construcción de la paz, la defensa de los derechos humanos y los valores de la democracia.

5. Existe la necesidad de lograr más información, apertura y transparencia en lo tocante a las diversas misiones y actuaciones de cada establecimiento de enseñanza.

6. La autonomía es un requisito indispensable para que los establecimientos de enseñanza puedan cumplir con su cometido gracias a la calidad, la pertinencia, la eficacia, la transparencia y la responsabilidad social" (II Conferencia Mundial sobre la Educación Superior, 2009).

Lo que se quiere poner de manifiesto es que, en el marco de la CMES, especialmente en la primera de ellas, es donde la UNESCO más hincapié hizo en que el compromiso de la universidad lo es con un orden social fundado en la justicia social; lo es con mujeres y hombres, sin discriminaciones; lo es con el conjunto de la sociedad, no privilegiando sólo a un determinados grupos de interés; lo es con un modelo económico en el que el desarrollo humano es la meta, donde la economía está al servicio de las personas y, nunca, las personas al servicio de la economía.

Sin embargo, en su Estrategia para la Educación 2014-2021, es posible observar en la UNESCO cierta deriva mercantilista en cuanto a la labor de la educación superior que, por otro lado, no resulta casual. Como subraya en este sentido Rodrigues Dias, fue el año 2000 el momento en que la UNESCO y el Banco Mundial comienzan a alinear sus políticas educativas. Aquel año, durante la presentación del informe conjunto titulado La educación superior en los países en desarrollo: peligros y promesas, el entonces secretario general de la UNESCO, Sr. Koïchiro Matsuura declaró: "We have often, though perhaps not always, shared the same views on directions for its renovation and development" (Matsuura, 2000).

Ante esto y, pero sobre todo, ante el rumbo que la educación superior ha tomado recientemente en los documentos de la UNESCO, en los que no hay rastro de las valientes y oportunas propuestas de género realizadas en la Declaración de la CMES de 1998 o, ni tan siquiera, una mención a la Responsabilidad Social de la Educación Superior, resulta, cuando menos, oportuno considerar el temor de que el propósito de la UNESCO para abordar la elaboración de un instrumento jurídico internacional sobre educación superior (propuesta en otro tiempo formulada para proteger la libertad académica, la autonomía y la responsabilidad social) adopte, ahora, un contenido orientado a favorecer el interés del mercado global de la educación.

A pesar de este preocupante replanteamiento, se debe insistir y, así, recuperar el espíritu programático y transformador de las CMES, especialmente el de la primera reunión. Desde este foro es posible interpretar lo que debe considerarse como un compromiso socialmente responsable o, si se desea, una responsabilidad social de la universidad. Responsabilidad social que, por otro lado, 
y como señala Alma Herrera, "abarca un amplio conjunto de acciones y procesos que tienen como objetivo responder a las necesidades del entorno de manera oportuna, eficaz y con un alto sentido ético. Este planteamiento resulta de gran importancia, pues la globalización y la aplicación de modelos de desarrollo económico de carácter neoliberal han generado situaciones de urgencia social que demandan que las universidades coadyuven con respuestas innovadoras a afrontar la complejidad que hoy día sintetiza nuestra problemática nacional y regional. Tales respuestas innovadoras implican la articulación orgánica de las funciones sustantivas de las universidades y el replanteamiento de su papel en la solución de los complejos problemas de las sociedades del siglo XXI" (Herrera, 2009, p. 41).

La Universidad, asumiendo estos enfoques puede aspirar a recuperar su lugar en los procesos de creación de oportunidades de emancipación y transformación social. Las instituciones públicas universitarias deben dejar de ser parte de los problemas para convertirse, definitivamente, en parte de las soluciones. Empleando las palabras de Noam Chomsky se podría decir que "la vida universitaria no sólo debería ser liberadora para todos sus participantes, sino que también debería tener un impacto en la sociedad en general. En un sentido profundo, el papel social e intelectual de la universidad debería ser subversivo en una sociedad sana" (Chomsky, 2002, p. 132)

Por consiguiente, haciendo pleno el derecho de «autonomía con responsabilidad social» será posible recuperar, como Boaventura do Sousa Santos señala, la legitimidad institucional y social perdida (Santos, 2005, p. 47).

Se trata, de recuperar el espíritu perdido de la universidad clásica, aquel que José Luís Sampedro identifica con el saber, el conocer y el aprender (Sampedro, 2011).

Conseguir esto, además de hacer de la Universidad una institución comprometida con principios y valores éticos transformadores - aquellos que quedaron fuera del enfoque clásico de la universidad y que han sido preteridos por la reforma neoliberal-, requiere un cambio de paradigma en la educación superior y en las Universidades públicas, requiere también ofrecer un nuevo enfoque para la responsabilidad social de la universidad. Así frente al carácter economicista de la educación superior, donde las personas están al servicio del desarrollo y el crecimiento económico, se debe proponer otro en el que las personas y su desarrollo se convierte en la prioridad.

Este enfoque, conocido como "enfoque del desarrollo humano» enfatiza, como Alejandra Boni y Des Gasper señalan, los siguientes aspectos: “Una pluralidad de valores, no sólo los valores de la utilidad económica. Una preocupación y solidaridad por el ser humano; al igual que en la tradición de los derechos humanos, la referencia son todos los seres humanos, en cualquier parte del mundo, y en particular aquellos que se vean afectados por nuestras acciones. La normalidad y la centralidad de las interconexiones: los efectos colaterales de los mercados implican que los cálculos del mercado son inadecuados, aunque sólo utilicemos el valor de la utilidad económica" (Boni Aristizábal y Gasper, 2011, p. 99).

Impulsar este cambio de paradigma, asumir el enfoque del desarrollo humano, lleva, a su vez, a replantear el êthos universitario, dotándolo de una eticidad - fundada en la ética cosmopolita de mínimos (Rueda López y Sanchis Vidal, 2016) y en la función ética de la educación superior señalada en la Declaración de la CMES de 1998- en cada uno de sus ámbitos específicos: la gestión 
de la institución, la docencia, la investigación y la extensión. Esta investigación se focaliza, como ya ha sido indicado, precisamente en el primero de estos ámbitos.

\section{METOdOLOGÍA.}

Las técnicas científicas empleadas en esta investigación han sido el método analíticosintético, el análisis normativo y la perspectiva de género.

El empleo del método analítico-sintético ofrece la posibilidad, en primer término, de dividir el objeto de estudio en los principales elementos que lo componen para, a partir de los mismos, abordar su análisis y poder determinar sus características y particularidades. Al mismo tiempo, la síntesis permite integrar, cada una de estas particularidades y características, desde una perspectiva integral e interrelacionada lo que lleva a comprender el objeto de estudio en su dimensión plena y conjunta.

En cuanto al método de análisis normativo empleado, su aplicación viene justificada por la necesidad de determinar las dinámicas jurídicas que, desde el ámbito internacional y nacional, han permitido dictar las normas que han concretado los valores y principios éticos y que, a su vez, han hecho posible el funcionamiento del orden social y político hegemónico en cada momento, particularmente en el ámbito de la educación.

Por último, la transversalización de la perspectiva de género como método científico, en las metodologías anteriores y, por tanto, en toda la investigación, hará posible el análisis y la comprensión de las realidades y características particulares de mujeres y hombres de manera diferenciada, atendiendo, a su vez, la relación y dependencia entre los géneros. Esto no sólo permito desentrañar las dinámicas políticas, normativas, económicas y sociales que han permitido la perpetuación de la violencia y la discriminación de las mujeres, sino, además, establecer las estrategias y tácticas necesarias para erradicar las mismas.

Las fuentes documentales - primarias y secundarias - consultadas, especialmente las bibliográficas, son numerosas, lo que ha dado lugar a una importante revisión de las mismas. A pesar de no ser esta una investigación positivista, no se ha eludido la consulta de doctrina, normativa y jurisprudencia. Se ha cuidado plantear la argumentación sociológica, política y filosófica a la vez que presentar los marcos normativos de referencia en cada uno de los aspectos abordados.

\section{RESULTADOS Y DISCUSIONES.}

\subsection{Financiación universitaria.}

Desde la consagración del Consenso de Washington como el marco rector de la vida económica y política de la globalización neoliberal, los Estados han reducido la financiación pública de un conjunto de derechos sociales, entre ellos la educación. Como se ha tratado de demostrar a lo largo del apartado anterior, en el caso de la educación superior, ha tenido unas causas y consecuencias particulares. En el caso de la autonomía universitaria, la reforma neoliberal ha llevado a las universidades a una nueva instancia del viejo modelo universitario napoleónico, en el 
que el control y el poder del Estado ha sido sustituido por el control y el poder de los mercados. Las universidades, durante este tiempo, han visto como el espacio de su libertad y de su autonomía ha ido quedando reducido al ámbito de la «sabiduría convencional». Dentro de ella, toda libertad es posible, fuera nada existe.

Recuperar un escenario pleno de libertad y autonomía, desde el que poder dar sentido a la «autonomía con responsabilidad» invocada requiere, por tanto, la protección pública. Esta es la parte más elemental de la responsabilidad que los Gobiernos deben asumir en cuanto a la educación superior y se debe concretar en un marco de financiación que garantice el carácter público y de la misma. En este sentido, en el caso de España, una «Ley de financiación de las universidades públicas» debería constituir el instrumento jurídico adecuado desde el que garantizar a las universidades públicas el acceso a los recursos financieros suficientes que les permitan desarrollar su misión y sus funciones. Sólo la financiación pública puede garantizar la «autonomía con responsabilidad» de la universidad.

Pero además este marco de financiación debe garantizar a todas las personas el acceso universal a la educación superior de manera equitativa y garantizando la igualdad de oportunidades.

Sin embargo, a partir de que en 1986 Bruce Johnstone desarrollase el concepto de «distribución del gasto» o "gasto compartido» la financiación de la Universidad pública ha tomado otro rumbo. Con esta idea Johnstone introdujo un modelo según el cual la financiación del coste de la educación superior debía repartirse entre el sector público, las familias, el alumnado egresado y el sector privado (Johnstone, 2011, pp. 115-117).

En 1994 el Banco Mundial adoptaba esta tesis como una de las reformas propuestas y uno de los requisitos Ilamados a garantizar la calidad y la eficiencia de los sistemas de educación superior (Banco Mundial, 1995, p. 44).

Así, el "gasto compartido» o la diversificación del financiamiento (como el propio Banco Mundial llamó a las tesis de Johnstone) se convertiría desde entonces en la corriente dominante entre los modelos diseñados (hasta cuatro en función de la proporción de gasto que cada parte debe asumir) para financiar la educación superior (Pérez Esparrells y Gómez Sancho, 2010, pp. 35-36). Favoreciendo que la participación pública en la financiación del servicio de educación superior haya pasado del 86\% en el año 1995 al 70\% en el 2014 en el marco de los países de la OCDE (Organisation for Economic Co-operation and Development, 1998, p. 102; 2017, p. 200).

Por su parte, la Asociación Europea de Universidades, lineada con los postulados del «coste compartido" ha considerado que este representa la oportunidad que las universidades tienen de diversificar sus ingresos, garantizando, de esta manera, su sostenibilidad financiera como institución y la de sus actuaciones en su misión académica e investigadora. Para esto, señala la misma entidad, resulta del todo necesario que los gobiernos eliminen todas aquellas barreras legislativas existentes que impiden que las universidades puedan desarrollar su potencial en cuanto a la generación de ingresos (European University Association (EUA), 2011, p. 44 y 85).

Por otro lado, en cuanto a los rechazos que genera la sustitución de la financiación pública por la privada se deben tomar en cuenta opiniones como las de Francisco Fernández Buey quien, al referirse a la autonomía universitaria, señaló que ésta ha dejado de ser independencia del poder 
político para convertirse en dependencia del poder político-industrial (Fernández Buey, 2009, p. 234). Desde el ángulo de la misión social que la universidad debe tener, Martín Rodríguez Rojo señala que "abandonar la financiación de la universidad en manos privadas implicaría dejar de considerarla como institución pública, al servicio de [la ciudadanía]. Sólo lo consentiría un Estado dispuesto a servir a una globalización de mercados y no a una globalización social" (Rodríguez Rojo, 2000, p. 91).

La financiación pública es una herramienta política que garantiza la autonomía universitaria y, por tanto, su libertad, su reducción o desaparición provoca que la misión y funciones de la universidad se vinculen cada vez más a las necesidades y demandas del mercado. De esta manera la institución universitaria pierde autonomía, ya que la lógica de servicio público que su praxis debe seguir, va siendo reemplazada por la lógica del mercado. En esta última, los beneficios financieros se sitúan por encima del beneficio social, o, en otras palabras, la rentabilidad social de la universidad queda relegada en relación a su rentabilidad económica. Este es el caso de uno de los pilares de la universidad, la producción científica que, en el contexto de la "sociedad del conocimiento" y como será abordada más adelante, queda reducida exclusivamente al valor que ésta alcance en el mercado.

Las universidades, después de ver recortada su financiación deben recurrir al capital privado para salvar sus necesidades de financiación, lo que no sólo repercute en una mercantilización de la Academia, sino que además acerca la universidad pública a la privada "trasformando la universidad, en su conjunto, en una empresa, una entidad que no produce solamente para el mercado sino que produce en sí misma como mercado, como mercado de gestión universitaria, de planes de estudio, de diplomas, de formación de docentes, de evaluación de docentes y estudiantes" (Santos, 2005, p. 17).

Con todo, lo que es posible observar es que la relación universidad-sociedad, en la que la educación y su carácter estaban determinados por las expectativas de toda la sociedad, es, de manera progresiva y a través de los mecanismos de financiación, suplantada por una relación universidad-empresa en la que las instituciones universitarias sufren una transformación que la incorpora a los circuitos empresariales y mercantiles de la sociedad capitalista actual, ámbito en el que el bien común que representa la educación superior y la universidad es preterido frente a los intereses empresariales.

\subsection{La igualdad de género.}

En cuanto al primero de estos principios, las universidades deben dejar de ser esos ámbitos profundamente masculinizados donde las mujeres son, aún en determinadas ocasiones, invisibles para muchos estándares académicos. Las mujeres representan el $50 \%$ de la población, por tanto, las Universidades deben convertir, definitivamente, todos sus ámbitos, institucional, docente, investigador y de gestión, en fiel reflejo de esta realidad. No es suficiente con que las mujeres sean mayoritarias en las aulas, también han de serlo conforme ascendemos hacia la cúspide académica, ámbitos donde su presencia dista mucho de ser la correspondiente a una institución igualitaria.

En el caso de España, las Universidades no sólo deben cumplir lo dispuesto en relación a la promoción de la igualdad entre mujeres y hombres en la Ley Orgánica de Universidades, sino que 
además no pueden ser ajenas, bajo ningún concepto, a lo dispuesto en la Ley Orgánica 3/2007, de 22 de marzo, para la igualdad efectiva de mujeres y hombres, particularmente lo dictado en sus artículos 23, 24 y 25, pero también lo recogido en el artículo 15.

En virtud de lo dispuesto en este último articulo, las universidades públicas quedan sujetas a la transversalización del principio de igualdad de trato entre mujeres y hombres, integrando el mismo, de forma activa, en la definición de políticas y estrategias universitaria. En otras palabras, las políticas de género en la Universidad pública deben dejar de convertirse en estas cuestiones reservadas a las unidades de igualdad, pasando a formar parte, de manera transversal, de la vida académica con el fin de alcanzar una representación equilibrada entre mujeres y hombre en todos los ámbitos universitarios.

Esta es la estrategia más acertada para, a su vez, poder llevar a cabo lo dispuesto en el artículo 23 de la misma Ley, según el cual "el sistema educativo incluirá, dentro de sus principios de calidad, la eliminación de los obstáculos que dificultan la igualdad efectiva entre mujeres y hombres y el fomento de la igualdad plena entre unas y otros". Disposición que, por otro lado, fue señalada como estratégicamente prioritaria en el informe El gobierno de la educación superior en Europa. Políticas, estructuras, financiación y personal académico publicado por EURYDICE (2008).

Dicho esto, la eliminación de todas las barreras de género que dificultan la promoción y el desarrollo de la carrera académica de las mujeres, provocando escenarios de "segregación vertical» en las universidades, se debería haber convertir, años atrás, en una política estratégica de primer orden en el esfuerzo para alcanzar mayores cotas de democracia institucional en las universidades públicas.

Sin embargo, tan sólo tres años después de aquella declaración estratégica, EURYDICE reconocía en su informe Diferencias de género en los resultados educativos: medidas adoptadas y situación actual en Europa, que tan "sólo cerca de un tercio de los países analizados cuentan con políticas o proyectos dirigidos a combatir la segregación vertical en la educación superior" (EURYDICE, 2011, p. 105).

Más allá del nivel analítico que nos ofrecen los informes de EURYDICE, en el plano político, el Parlamento Europeo, a través de su Resolución, de 9 de septiembre de 2015, sobre las carreras profesionales de las mujeres en los ámbitos científico y académico y los techos de cristal existentes, señala que "es necesario introducir cambios en las instituciones a fin de vencer los obstáculos a la igualdad de género, especialmente en lo que respecta a la segregación vertical y a la participación de las mujeres en los comités con poder de decisión" (Unión Europea, 2015).

En el caso de España, los planes de igualdad impulsados en las Universidades públicas constituyen herramientas que favorecen dinámicas relevantes para alcanzar el propósito, entre otros, de reducir la "segregación vertical» en las Universidades y es posible, a su vez, reconocer los esfuerzos y avances realizados desde su implantación. Sin embargo, no es menos cierto considerar, a la luz de los resultados obtenidos por diversas investigaciones, que los mismos parecen insuficientes.

Para Ion, Durán y Bernabeu (2013) "todavía, a inicios de la segunda década del siglo XXI, el profesorado participante en nuestra investigación percibe una situación de desigualdad de género 
persistente en sus lugares de trabajo. Las razones que sustentan dicha desigualdad son similares a las halladas por los estudios realizados durante la década de los noventa y con anterioridad" ( $p$. 130).

Quizás uno de los ámbitos más visibles y evidentes de la persistencia de estas situaciones de desigualdad entre géneros, sea el de los órganos de gobierno y de gestión de las Universidades públicas españolas. En este sentido, como señalan en su investigación Rekalde y Cruz (2017), en las 50 Universidades públicas españolas los cargos unipersonales por designación (Secretaría general, Gerente y Vicerrectores/as) que son ocupados por mujeres alcanzan el 40'91\% y tan sólo en tres de estas Universidades, el 6\%, el cargo de Rector/a es ostentado por una mujer. Como Rekalde y Cruz concluyen "si bien la distancia numérica entre hombres y mujeres es cada vez menor, la persistencia de ámbitos y áreas estereotipados que actúan en paralelo es todavía apreciable" (p. 141).

Con todo, parece que el género, de nuevo citando a Ion, Durán y Bernabeu (2013) "sigue siendo un elemento de desigualdad a pesar de las medidas universitarias que se están aplicando. Podríamos considerar que existe una brecha entre lo "oficialmente escrito» y lo "realmente vivido»" (p. 136).

Señalar que reclamar, como así se esta haciendo para la Univerisdad, el sentido de la democracia paritaria implica revisar, como así hace Octavio Salazar (2014) el concepto de masculinidad hegemónica. En este sentido, existe la necesidad "no sólo de que haya más mujeres tomando decisiones, sino también que se incorporen nuevos métodos de toma decisiones y de resolución de conflictos, nuevos criterios de valoración de méritos y nuevas palabras para nombrar realidades que antes eran invisibles. Estos objetivos han de obligar a una transformación no sólo de las instituciones y de los procesos decisorios, sino también de los instrumentos que en democracia canalizan la representación política" (p. 627).

Sin embargo, como señalan Donoso-Vazquez y Velasco-Martínez (2013), "la perspectiva de género parte de la teoría feminista sin la cual el concepto está vacío de contenido y se convierte en un vocablo neutro, contemporizador de un orden social injusto, jerarquizado y desigual" (p. 84): el orden social patriarcal. Esto fundamenta el hecho de que las teorías feministas deban pasar a formar parte de los planes de estudio académicos cuanto antes.

Junto a todo lo anterior, la universidad debe constituirse como el espacio idóneo para garantizar el derecho que cada persona tiene a elegir quien quiere ser (Vasquez y Lajud, 2016).

Esta idea resulta útil para introducir el segundo de los principios invocados para profundizar los procesos de democratización universitaria; el respeto a la diversidad y la pluralidad de cosmovisiones.

\subsection{El respeto a la diversidad y la pluralidad de cosmovisiones.}

La Declaración de la CMES de 1998 señaló, en su artículo primero, que la misión de la educación superior, entre otras, deberá "contribuir a comprender, interpretar, preservar, reforzar, fomentar y difundir las culturas nacionales y regionales, internacionales e históricas, en un contexto de pluralismo y diversidad cultural" (Conferencia Mundial sobre la Educación Superior, 1998). 
Sin embargo, el principio que se quiere invocar va más allá de lo establecido en esta Declaración, circunscrito al ámbito cultural. En este sentido, apelar al respeto a la diversidad y la pluralidad de cosmovisiones es, a su vez, hacerlo directamente a la tercera de las características de la ética cosmopolita de mínimos, el pluralismo de proyectos humanos y cosmovisiones (Rueda López y Sanchis Vidal, 2016). Desde esta instancia, además de la cultura, se incluyen las propias opciones individuales de las personales, sus creencias y cosmovisiones, sagradas o profanas, su diversidad afectiva y sexual, en definitiva, se trata de asumir un pluralismo cosmopolita que vindique la diferencia, y en él ésta presente la laicidad como valor.

Como indica Tamayo Acosta (2012), la laicidad "constituye el espacio político, el marco jurídico y el horizonte ético más adecuados para el reconocimiento y el ejercicio de los derechos humanos y de las libertades de conciencia, de expresión, de asociación y de religión, así como para el reconocimiento de las ideologías, los sistemas de creencias, y los proyectos utópicos que se expresen y defiendan pacíficamente" (p. 266).

Como se reclama en la ética cosmopolita en cuanto a la construcción de una sociedad laica y plural, en este objetivo deben concurrir el conjunto de instituciones públicas (Rueda López y Sanchis Vidal, 2016).

Es este el espíritu, aunque quizás expresado de manera más simplificado, que se recoge en el preámbulo de la Ley Orgánica 4/2007, de 12 de abril, por la que se modifica la Ley Orgánica 6/2001, de 21 de diciembre, de Universidades, cuando señala que "el reto de la sociedad actual para alcanzar una sociedad tolerante e igualitaria, en la que se respeten los derechos y libertades fundamentales y de igualdad entre hombres y mujeres, debe alcanzar, sin duda, a la universidad".

Por tanto, en un tiempo donde la «sabiduría convencional» o el pensamiento único neoliberal ha conseguido imponer un universalismo en el que la heteronormatividad castiga la diferencia, la universidad debe asumir, desde los valores del pluralismo cosmopolita, la responsabilidad social de ofrecer espacios donde las diferencias entre grupos sociales sean reconocidas y protegidas como expresión última de una sociedad plural.

En este sentido Pérez Tapias (2007) señala que "frente a toda pretensión de monopolio acerca de la verdad o en cuanto a concepciones de lo bueno desde la que se quisiera imponer unilateralmente lo que para la sociedad en su conjunto ha de ser justo, el vínculo inseparable de pluralismo y laicidad es garantía de la coherencia democrática que necesita una "sociedad abierta»" (p. 292).

Sólo desde el respeto pleno a la diversidad y la pluralidad de cosmovisiones las universidades podrían ser capaces de ofrecer una alternativa al pensamiento hegemónico, y a la "sabiduría convencional».

Pluralismo y diversidad que, por tanto, avanzan conjuntos como valores indisociables. Como señala Fariñas Dulce (2014) "el concepto del pluralismo no se debe contraponer a la existencia de conceptos universales, ya que cada cultura o cosmovisión tiene sus propios fundamentos o cimientos universales 0 , incluso, cada universo cultural es una imagen del mundo en su totalidad, incomprensible para otros. Por eso la convivencia entre culturas suele ser complicada y requiere un esfuerzo recíproco por no imponer condiciones absolutas a la convivencia" (p. 35). 
Construir una sociedad desde el pluralismo cosmopolita lleva implícito la eliminación de todas aquellas formas y actitudes, sociales e individuales, que dificultan la convivencia. Por tanto, combatir la discriminación, en cualquiera de las representaciones que ésta pueda adquirir (por género, por edad, por origen étnico, por creencias religiosas, por la orientación o identidad sexual), debería constituir una práctica de primer orden en la praxis universitaria para la protección de las diferencias y de cada uno de los grupos de riesgo, sobre todo a la luz de los datos que arrojan diversos estudios e informes.

En España, como reveló el estudio titulado Los perfiles de la discriminación en España, "la experiencia de haber sido discriminado o discriminada en algún momento de la vida no es demasiado extraña y el 31,5\% de la población declara haber sido discriminada muchas veces o alguna vez. Con un periodo de referencia más preciso, el 15,8\% declara haber sido discriminado en los últimos 12 meses" (Zugasti, 2014, p. 64).

En este estudio, como se presenta en la tabla 1, se identifican cómo afecta cada representación de la discriminación a cada grupo de riesgo.

Tabla 1. Incidencia, en porcentajes, de cada tipo de discriminación en la población general y en cada grupo de riesgo.

\begin{tabular}{|c|c|c|c|c|c|c|c|}
\hline & \multicolumn{3}{|c|}{$\begin{array}{c}\text { Frecuencia del } \\
\text { sentimiento de } \\
\text { discriminación a lo largo } \\
\text { de la vida }\end{array}$} & \multirow{2}{*}{$\begin{array}{l}\text { Sentimiento de } \\
\text { discriminación } \\
\text { en los últimos } \\
12 \text { meses }\end{array}$} & \multirow[t]{2}{*}{$\begin{array}{l}\text { Definición del } \\
\text { grupo de riesgo }\end{array}$} & \multicolumn{2}{|c|}{$\begin{array}{c}\text { Relación con el grupo de riesgo } \\
\text { № de personas afectadas del } \\
\text { grupo de riesgo/№ de personas } \\
\text { del grupo de riesgo }\end{array}$} \\
\hline & Muchas & Algunas & Total & & & $\begin{array}{l}\text { En alguna } \\
\text { ocasión }\end{array}$ & $\begin{array}{c}\text { En los últimos } 12 \\
\text { meses }\end{array}$ \\
\hline Sexo & 2,5 & 8,9 & 11,4 & 3,8 & Mujer & 19,6 & 6,6 \\
\hline $\begin{array}{l}\text { Nacionalidad u } \\
\text { origen étnico }\end{array}$ & 2,1 & 7,3 & 9,3 & 4,2 & $\begin{array}{c}\text { Con origen } \\
\text { familiar personal } \\
\text { extranjero }\end{array}$ & 34,2 & 18,2 \\
\hline $\begin{array}{l}\text { Orientación } \\
\text { sexual }\end{array}$ & 0,3 & 1,1 & 1,4 & 0,3 & $\begin{array}{l}\text { Homosexual o } \\
\text { bisexual }\end{array}$ & 13 & 4,8 \\
\hline \multirow[t]{2}{*}{ Edad } & \multirow[t]{2}{*}{2,2} & \multirow[t]{2}{*}{8} & \multirow[t]{2}{*}{10,3} & \multirow[t]{2}{*}{5,4} & $\begin{array}{l}\text { Entre } 16 \text { y } 29 \\
\text { años }\end{array}$ & 13,5 & 7,6 \\
\hline & & & & & Mayores de 65 & 2,7 & 1,2 \\
\hline $\begin{array}{l}\text { Creencias } \\
\text { religiosas }\end{array}$ & 0,3 & 3 & 3,3 & 1,2 & & & \\
\hline $\begin{array}{l}\text { Pocos recursos } \\
\text { económicos }\end{array}$ & 1,5 & 5,3 & 6,8 & 3,1 & Pobreza subjetiva & 13,8 & 7,7 \\
\hline Discapacidad & 0,3 & 1,6 & 1,8 & 0,7 & & & \\
\hline $\begin{array}{l}\text { Enfermedad } \\
\text { crónica }\end{array}$ & 0,2 & 0,8 & 1 & 0,3 & & & \\
\hline $\begin{array}{l}\text { Por sus ideas } \\
\text { políticas }\end{array}$ & 1 & 4,5 & 5,4 & 2,6 & & & \\
\hline
\end{tabular}


Como se puede observar, la discriminación por motivos de sexo se constituye como la principal caracterización de las dinámicas de discriminación. Así, el 2,5\% y el 8,9\% de la población reconoce haber sido discriminada muchas veces o alguna vez por este motivo. en total, por motivos de sexo, el $11,4 \%$ de la población ha sido discriminada a lo largo de su vida.

Frente al pensamiento de que este dato está fundamentado en el hecho de que las mujeres representan el $50 \%$ de la población, la relación del número de personas afectadas por la discriminación entre el número de personas total que componen dicho grupo revela que el 19,6\% de las mujeres han sido víctimas de discriminación por motivos de sexo, y que el sexo se sitúa, tras la nacionalidad o el origen étnico (34,2\%), como la segunda de las formas que caracterizan a la discriminación, seguida de las creencias religiosas $(13,9 \%)$, la pobreza $(13,8 \%)$, la juventud $(13,5 \%)$ y la orientación o identidad sexual (13\%).

Pero además, si se atiende al concepto de discriminación múltiple se comprueba que "las personas que manifiestan haber vivido a lo largo de su vida simple, doble o múltiple discriminación son en su mayoría mujeres ( $51,3 \%, 59,9 \%$ y $58,4 \%$, respectivamente), también de $30-44$ años (38\%, $35,4 \%$ y $35,6 \%$ respectivamente), pero, en el caso de la discriminación múltiple, se constata que hay 12 puntos porcentuales más de jóvenes (18-29 años) con 3 o más discriminaciones, en relación a las personas sin discriminación (16,3\% frente a $28,3 \%)$. De tal forma que observamos que, si bien hay más mujeres con doble o múltiple discriminación, las personas jóvenes aumentan a nivel porcentual conforme se van acumulando más discriminaciones" (Martínez, 2014, p. 117).

Después de todo, lo que se desea argumentar es que, en el actual orden social, a pesar de los avances, el recorrido para alcanzar un escenario de igualdad, pluralidad y de respeto hacia la diferencia es aún largo. Un camino en el que la educación, en general, y la Universidad, en particular, poseen una importante responsabilidad en aras de acelerar los procesos de transformación social.

\subsection{El diálogo con los grupos sociales y de interés de la universidad.}

La llamada a establecer nuevos lazos entre la Universidad y la sociedad ha sido una constante desde hace décadas. Las Universidades y la sociedad tenían la necesidad perentoria de que las primeras abandonasen las "torres de marfil» en las que habían estado encerradas y pasaran a formar parte activa de las dinámicas de la segunda. Como desde muchas instancias se ha señalado, se trataba de adoptar un nuevo contrato social entre la universidad y la sociedad (Benneworth y Jongbloed, 2010; Jasanoff, 2009; Neave, 2006).

El concepto de pertinencia universitaria y el ideario de la Responsabilidad Social Universitaria habían sido concebidos para que las universidades transitaran desde su encierro hasta el lugar en el que ocupar ese nuevo protagonismo social: aspiraban a ser, sino todo, parte de ese nuevo contrato social.

Sin embargo, como también de alguna manera se ha venido sosteniendo, ambos conceptos más que dar forma o ser parte de ese contrato social, han servido para justificar un determinado modelo de universidad destinado, en última instancia, a primar unos intereses frente a otros. 
Se afirmó, sobre todo en relación a la pertinencia universitaria, que ésta, como consecuencia de su carácter de "significante vacío», había adoptado la forma economicista propia de la corriente que goza del privilegio de la hegemonía, en este caso el neoliberalismo.

Por tanto, desde la línea argumental que guía esta investigación, profundizar la democracia institucional como factor insoslayable de la "autonomía con responsabilidad», es condición necesaria para hacer de la pertinencia social una realidad y de la responsabilidad social de la universidad una práctica inclusiva. La universidad debería, como sostiene Santos (2005), "ser permeable a las demandas sociales, especialmente aquellas originadas en grupos sociales que no tienen el poder para imponerlas" (p. 62).

En España, el artículo 14.1 de la LOU determina que los Consejos Sociales de las universidades son los órganos "...de participación de la sociedad en la universidad, y deben ejercer como elementos de interrelación entre la sociedad y la universidad".

Sin embargo, esta participación, aparentemente amplia, queda reducida cuando, en virtud del apartado segundo del mismo artículo, se antepone, a cualquier otra, los siguientes fines de los Consejos Sociales: "la supervisión de las actividades de carácter económico de la universidad y del rendimiento de sus servicios y promover la colaboración de la sociedad en la financiación de la universidad". Competencias que se amplían y desarrollan a lo largo de diversos títulos de la propia LOU, como el XI sobre el régimen económico y financiero de las Universidades públicas, así como otras relacionadas con nombramientos de cargos directivos de la universidad.

Finalmente, la LOU delega, en virtud del apartado tercero del mencionado artículo, en las Comunidades Autónomas regular “...la composición y funciones del Consejo Social y la designación de sus miembros de entre personalidades de la vida cultural, profesional, económica, laboral y social...".

Esta delegación conlleva, en este momento, aplazar el análisis detallado de la diversa legislación autonómica que regula los consejos sociales. En cualquier caso, aún asumiendo que resulta lejano en el tiempo, es posible considerar algunos de los resultados y de las conclusiones del estudio publicado por la Conferencia de Consejos Sociales de las Universidades Públicas Españolas (2010) en cuanto a los elementos comunes de la legislación autonómica reguladoras de los Consejos Sociales de las Universidades públicas españolas.

Este informe puso de manifiesto que entre las competencias comunes de los consejos sociales se encuentran las relacionadas con:

- La planificación y el desarrollo institucional: plan estratégico, convenios de financiación y contratos programa, creación de entes instrumentales, implantación de nuevas titulaciones.

- La gestión económica: el presupuesto, aprobación de las cuentas anuales de la universidad y de sus entidades dependientes, la programación plurianual, operaciones de endeudamiento, adquisición y/o desafectación de bienes, supervisión de las actividades económicas de la universidad, precios públicos. 
- La gestión de los servicios: supervisión del funcionamiento de los servicios, personal de administración y servicios, personal docente e investigador, nombramiento y cese del gerente, convenios y contratos.

- Las relaciones con la sociedad: promoción de la colaboración de la sociedad en la financiación de la universidad, plan anual de relaciones de la universidad con el entorno, designación de vocales del Consejo Social como miembros del Consejo de Gobierno.

- La gestión académica: normas de permanencia, creación, modificación y supresión de centros; y adscripción de centros e institutos, becas y ayudas.

Por otro lado, en cuanto a la composición de los consejos sociales, a la luz de la información publicada en las páginas web de los mismos se pueden obtener dos conclusiones esenciales: 1) entre las personas que forman parte de sus órganos, existe una mayor representación de hombres que de mujeres, hasta el extremo que, en el significativo caso de la Conferencia de Consejos Sociales entidad que representa al conjunto de los consejos sociales de las universidades españolas ninguno de sus cargos unipersonales es ocupado por una mujer y tan sólo tres, del total de doce personas que forman parte de su Comité ejecutivo, son mujeres. 2) La representación de las organizaciones sociales se limita, en la gran mayoría de los casos, a personas que ocupan el papel de vocal designadas a propuesta de las organizaciones empresariales y sindicales más representativas en cada territorio.

Con todo, lo que se observa es que, en cuanto a las competencias, éstas están relacionadas con el control de la labor universitaria desde una perspectiva, fundamentalmente, gestora. Cuando esto no es así, existe una clara tendencia a vincular la labor de los consejos sociales con los sectores económicos y productivos. Pero incluso en el caso de asumir éstas como las prioridades que los consejos sociales deben ejercer, lo relevante es que la composición de los mismos no es una representación fiel de la sociedad, ni en cuanto a la proporción de mujeres, ni en cuanto a la realidad de la sociedad civil organizada. En este último caso, como señala Galcerán Huguet (2010), "en la medida, también, en que la universidad debe estar cada vez más cerca de la sociedad (el mercado) se potencia que sus representantes más destacados estén en los Consejos Sociales y así encontramos en ellos a los grandes empresarios de los grupos hegemónicos" (p. 19). Encontramos, así, como muchos consejos sociales son presididos por personas estrechamente ligadas a intereses empresariales, decisión, por otro lado, tomada desde instancias políticas.

Ante esta realidad no resulta extraño pensar que una de las propuestas clásicas del Círculo de Empresarios - uno de los más relevantes "think tank» económicos de España- sea, precisamente, que "los Consejos Sociales cumplan plenamente una función más cercana al consejo de administración [de una empresa, teniendo] la facultad de nombrar al Rector que asumirá las responsabilidades del gobierno actuando, así como Consejero Delegado de la Universidad" (Círculo de Empresarios, 2007, pp. 58-59).

Por tanto, lo que se pretende poner de manifiesto es que, en España, el modelo de participación de la sociedad en la universidad propuesto a través de los consejos sociales resulta, cuando menos, insuficiente. 


\section{CONCLUCIONES}

\subsection{Financiación universitaria}

El modelo de "gasto compartido» no parece garantizar los principios de equidad, ni ser el instrumento adecuado desde el que garantizar la financiación pública del sistema. Debería, por tanto, ser sustituido por otro en el que, en primer lugar, las familias no sean grabadas con tasas académicas y en el que el futuro de la juventud no quede hipotecado por cargas con entidades financieras, pero que, además, garantice la plena autonomía y libertad universitaria.

Explorar opciones vinculadas al nivel de renta de las personas físicas obtenidas durante su vida laboral como, por ejemplo, la creación de un impuesto progresivo para personas tituladas, puede constituir una opción - aún no llevada a la práctica en ningún sistema educativo- destinada a financiar el sistema público de educación superior garantizando mayores cotas de equidad (Salmi y Hauptman, 2005). Abordar el estudio sobre esta modalidad impositiva constituirá el objetivo de una investigación posterior.

Alcanzar este nuevo marco de financiación es difícil, no depende de las universidades, sino de instancias políticas. Mucho más cuando la educación en su conjunto, en el caso de España, lejos de ser una política de Estado, ha sido convertido en un campo de batalla de las diferentes opciones políticas. Eso sí, desde la universidad se puede educar a la ciudadanía y a las generaciones del futuro con un sentido crítico y libre desde el que reclamar estos derechos. Pero, además, se puede y se deben forjar alianzas sociales más amplias desde las que defender y hacer valer el derecho a una educación superior libre y autónoma.

\subsection{La igualdad de género.}

Resulta necesario que desde la noción de universidad comprometida se adquiera, de manera insoslayable, un compromiso efectivo de la Universidad con el principio de igualdad de trato entre mujeres y hombres que haga de las instituciones universitarias espacios inclusivos, capaces de deslegitimar la heteronormatividad imperante favorecedora de desigualdades y violencia hacia lo diferente.

Este compromiso institucional es posible mediante medidas tan pertinentes como, por ejemplo, aplicar estadísticas anuales desagregadas por sexo en todas y cada una de las actuaciones universitarias, lo que facilitaría los análisis y diagnósticos para mostrar la realidad y, quizá, ayudaría a implementar las medidas oportunas para cerrar las brechas de género (Sanchis Vidal, 2015).

Por otro lado, asumir las políticas de género e igualdad desde el ámbito de los órganos de gobierno y representación de las universidades, sin duda, llevaría a un nivel superior la transversalización de las mismas en la vida académica (Mentado Labao, Montané López, y Olivé Ferrer, 2012). En este sentido, la propuesta de crear, más allá de las unidades de igualdad, concretamente vicerrectorías para la promoción de la igualdad, supondría un paso significativo y un compromiso institucional de primer nivel.

Desde esta instancia, se deberían impulsar, como subrayan Donoso-Vázquez y GarcíaFerrando (2014), procesos de empoderamiento que tiendan a aclarar: "a) cómo se abordan las 
oportunidades de las mujeres en el acceso a los recursos ideológicos, la participación económica y política y la toma de decisiones; b) cómo se promueve la toma de conciencia del poder individual y colectivo y el desarrollo de la dignidad como persona; c) cuáles son las barreras que la opresión internalizada de las mujeres hace que el acceso y ejercicio del poder sea discriminatorio y desigual" (p. 273).

\subsection{El respeto a la diversidad y la pluralidad de cosmovisiones.}

Las Universidades públicas, por tanto, en virtud de su mandato social, deberían asumir un compromiso y tareas concretas a través de sus funciones sustantivas en este camino. En relación a este compromiso institucional, la creación e impulso de, por ejemplo, un observatorio universitario para el fomento del pluralismo, la diversidad y la igualdad, dependiente de las vicerrectorías para la promoción de la igualdad antes propuesta y de similares características al Observatorio Estatal de la Convivencia Escolar podría ser considerada una iniciativa idónea en el ámbito universitario de lo que se desee reflejar con esta propuesta. que pudiera poner en marcha muchas de las recomendaciones y propuestas de los informes citados con anterioridad, sería algo más que deseable.

\subsection{El diálogo con los grupos sociales y de interés de la universidad.}

Es necesario, desde el carácter dialógico y democrático de la ética cosmopolita de mínimos, proponer un modelo de participación ciudadana en la universidad que transcienda al actual, para lo cual es necesario crear o redefinir los órganos de participación de la sociedad en las universidades.

Los consejos sociales o, en su caso, el órgano que cada universidad decida crear en virtud del artículo 2, letra c) de la LOU y de la idea de "autonomía con responsabilidad», deben responder, primero a los criterios de pluralidad y diversidad de la realidad social de su entorno, incorporando a todos los grupos y entidades que hasta ahora no han tenido capacidad de trasladar sus expectativas y demandas a las instituciones universitarias (Santos, 2005).

Se trata, de alguna manera, de sustituir la gestión corporativa de la universidad por aquella otra, de carácter social en la que activar, como indica Pinho de Oliveira (2016) “...mecanismos de operación y prestación de servicios a la ciudadanía, permitiendo la participación activa de la comunidad organizada. Y teniendo como premisa todos los valores superiores como la vida, la libertad, la justicia, la solidaridad, la igualdad, la democracia, la ética, la responsabilidad social y los derechos humanos" (pp. 17-18).

Por tanto, además de la educación en dinámicas y procesos de democracia participativa, y de la investigación y profundización teórica de la noción misma de democracia y de la participación ciudadana, tareas propias de las funciones docentes e investigadoras respectivamente, la universidad como institución debe poner en práctica mecanismos de democracia participativa.

En este sentido, sin negar la misión y competencias de los actuales consejos sociales, parece oportuno crear un órgano como, por ejemplo, los consejos universitarios de participación ciudadana. Este órgano tiene como propósito complementar la labor de los consejos sociales, pero situando la pertinencia social de la universidad en el centro del debate universitario mediante la participación de, además de la comunidad universitaria, organizaciones sociales de tipo poblacional 
(mujeres, jóvenes, mayores, colectivos LGTB o inmigrantes,) y sectorial (cultura, solidaridad, economía social y alternativa, ecologistas o vecinal). La participación y el empoderamiento de la sociedad civil organizada son dinámicas esenciales para avanzar en la agenda transformadora necesaria para la superación de los problemas humanos que se han venido diagnosticando (Veugelers y de Groot, 2014).

\section{BIBLIOGRAFÍA}

Banco Mundial. (1995). Enseñanza superior: las lecciones derivadas de la experiencia ([1* ed.). Washington D.C.: Banco Mundial.

Benneworth, P., y Jongbloed, B. W. (2010, 2010//). Who matters to universities? A stakeholder perspective on humanities, arts and social sciences valorisation. Higher Education, 59, 567588.

Boni Aristizábal, A., y Gasper, D. (2011, //). La Universidad como debiera ser. Propuestas desde el desarrollo humano para repensar la calidad de la Universidad. Sistema: Revista de ciencias sociales, 99-116.

Chomsky, N. (2002). La responsabilidad social de la universidad. In N. Chomsky, J. E. García-Albea, G. m. M. J. L., A. Domènech, y D. Comas d’Argemir (Eds.), Los limites de la globalización (pp. 126-139). España: Ariel.

Círculo de Empresarios. (2007). Una universidad al servicio de la sociedad. Madrid: Círculo de Empresarios.

Conferencia Mundial sobre la Educación Superior (1998). Declaración Mundial sobre la Educación Superior en el S. XXI: visión y acción. ED-98/CONF.202/CLD.49 (9 de octubre de 1998), disponible en: http://goo.gl/d1vZMC

Donoso Vázquez, T., y García-Ferrando, L. (2014). Empoderamiento de las mujeres y Educación Superior. In A. Teodoro y J. Beltrán, (Coords.) (Eds.), Sumando voces. Ensayos sobre educación superior en términos de igualdad e inclusión social (pp. 269-278). Buenos Aires: Miño y Dávila.

Donoso Vázquez, T., y Velasco Martínez, A. (2013). ¿Por qué una propuesta de formación en perspectiva de género en el ámbito universitario? Profesorado: Revista de curriculum y formación del profesorado, 17(1), 71-88.

European University Association (EUA). (2011). Financially sustainable universities II. Eropean universities diversifying income streams. Brussels: European University Association.

EURYDICE. (2008). El gobierno de la educación superior en Europa. Políticas, estructuras, financiación y personal académico. Madrid: Ministerio de Educación.

EURYDICE. (2011). Diferencias de género en los resultados educativos: medidas adoptadas y situación actual en Europa. Madrid: Ministerio de Educación.

Fariñas Dulce, M. J. (2014). Democracia y pluralismo: una mirada hacia la emancipación. Madrid: Dykinson.

Fernández Buey, F. (2009). Por una universidad democrática: escritos sobre la universidad y los movimientos universitarios (1965-2009). Mataró: El Viejo Topo.

Galcerán Huguet, M. (2010). La educación universitaria en el centro del conflicto. In F. Barchiesi, M. Bousquet, X. Ferreiro Baamonde, M. Galcerán Huguet, T. Herreros, J. Read, A. Ross, C. Vercellone, y J. Williams (Eds.), La universidad en conflicto: capturas y fugas en el mercado global del saber (1* ed ed., pp. 164 p.). Madrid: Traficantes de Sueños. 
Herrera, A. (2009). Responsabilidad social universitaria. In Global University Network for Innovation (GUNI) (Ed.), La educación superior en tiempos de cambio. Nuevas dinámicas para la responsabilidad social (pp. 40-41). Madrid: Mundi-Prensa.

II Conferencia Mundial sobre la Educación Superior (2009). Comunicado de la Conferencia Mundial sobre la Educación Superior: La nueva dinámica de la educación superior y la investigación para el cambio social y el desarrollo. ED.2009/CONF.402/2 (8 de julio de 2009), disponible en: http://goo.gl/IseLa8

Ion, G., Durán Bellonch, M. d. M., y Bernabeu Tamayo, M. D. (2013). El profesorado y su percepción sobre la igualdad de género en la universidad. Revista complutense de educación, 24(1 (Vol. 24)), 123-140.

Jasanoff, S. (2009). Implicaciones éticas, ambientales y sociales de la ciencia y la tecnología: retos futuros. In Global University Network for Innovation (GUNI) (Ed.), La educación superior en tiempos de cambio. Nuevas dinámicas para la responsabilidad social (pp. 76-80). Madrid: Mundi-Prensa.

Johnstone, B. (2011, 1 de marzo de 2016). Worldwide trends in financing higher education: a conceptual frameworl. Educación Superior y Sociedad, 16, 105-122.

Martínez, L. (2014). Discriminación múltiple. In Ministerio de Sanidad Servicios Sociales e Igualdad (Ed.), Los perfiles de la discriminación en España: Análisis de la Encuesta CIS-3.000. Percepción de la discriminación en España (pp. 115-130). Madrid: Ministerio de Sanidad, Servicios Sociales e Igualdad.

Matsuura, K. c. (2000). on the occasion of the ceremony to present the report: Higher Education in Developing Countries: Peril and Promise. Presentado en Ceremony to present the report: Higher Education in Developing Countries: Peril and Promise, Paris. on the occasion of the... Obtenido de http://goo.gl/AKHxJh.

Mentado Labao, T., Montané López, A., y Olivé Ferrer, M. C. (2012). Espacio de género. Una política en las universidades españolas a favor de la igualdad. In I. Vázquez Bermúdez, (Coord.) (Ed.), Investigación y género, inseparables en el presente y en el futuro: IV Congreso Universitario Nacional (pp. 1153-1168). Sevilla: Universidad de Sevilla.

Neave, G. (2006, 2006//). Redefining the Social Contract. Higher Education Policy, 19, 269-286.

Organisation for Economic Co-operation and Development. (1998). Education at a Glance 1998: OECD Indicators. Paris: OECD.

Organisation for Economic Co-operation and Development. (2017). Education at a Glance 2017: OECD Indicators. Paris: OECD.

Pérez Esparrells, M. d. C., y Gómez Sancho, J. M. (2010). Los rankings internacionales de las instituciones de educación superior y las clasificaciones universitarias en España: visión panorámica y prospectiva de futuro (1988-8767). Madrid: Obtenido de http://goo.gl/w2zHtP

Pérez Tapias, J. A. (2007). Del bienestar a la justicia: aportaciones para una ciudadanía intercultural. Madrid: Trotta.

Pinho De Oliveira, M. F. (2016, //). Los consejos comunales y la gerencia social comunitaria. Apuntes Universitarios, 6, 9-21.

Rekalde, I., y Iglesias, E. C. (2017). Las Mujeres en los Equipos de Gobierno en la Universidad Española. Un Estudio Discreto. Revista Internacional de Educación para la Justicia Social, 6(2), 129-146.

Rodrigues Dias, M.-A. (2010). ¿Quién creó este monstruo? Educación y globalización: sus relaciones con la sociedad1(2 (Vol. 1)). Retrieved from Vol. 1 núm. 2 (2010) website: https://goo.gl/zyg0iR 
Rodríguez Rojo, M. (2000, //). Sociedad, Universidad y Profesorado. Revista interuniversitaria de formación del profesorado, 79-99.

Rueda López, R., y Sanchis Vidal, A. (2016). Un modelo ético cosmpolita como propuesta para la convicancia intercultural. In Universidad de Córdoba (Ed.), Nuevas tendencias para la interculturalidad (1 1 ed., pp. 15-57). Córdoba: Universidad de Córdoba.

Salazar Benítez, O. (2014). La revisión de la masculinidad hegemónica como presupuesto de la democracia paritaria. In M. J. Terol Becerra y L. Jimena Quesada, (Dtores.) (Eds.), Tratado sobre protección de derechos sociales (pp. 609-629). Valencia: Tirant lo Blanch.

Salmi, J., y Hauptman, A. M. (2005). Mecanismos de asignación de recursos en la educación superior: tipología y evaluación. In Global University Network for Innovation (GUNI) (Ed.), La educación superior en el mundo 2006: la financiación de las universidades (pp. 60-83). Madrid: Mundi-Prensa.

Sampedro, J. L. (2011). José Luis Sampedro en diálogo con Olga Lucas. Autobiografía Intelectual: José Luis Sampedro. Presentado en Conferencias de la Fundación Juan March, Madrid. José Luis Sampedro en... Obtenido de http://goo.gl/LdAk3L.

Sanchis Vidal, A. (2014). Derechos humanos, democracia y género. De la ONU a la Universidad. In M. J. Terol Becerra y L. Jimena Quesada (Eds.), Tratado sobre protección de derechos sociales (pp. 631-655). Valencia: Tirant lo Blanch.

Sanchis Vidal, A. (2015). Interpretación jurídica, igualdad y género en los estudios de derecho. Aportaciones epistémicas y feministas. Revista General de Derecho Constitucional(21), 1-75.

Santos, B. d. S. (2005). La universidad en el siglo XXI. Para una reforma democrática y emancipadora de la universidad. Buenos Aires: Miño y Dávila.

Tamayo Acosta, J. J. (2012). Invitación a la utopía. Estudio histórico para tiempo de crisis. Madrid: Trotta.

Tünnermann Bernheim, C. (2008a). La Autonomía universitaria en el contexto actual. Universidades, 58(36), 19-46.

Tünnermann Bernheim, C. (2008b). La educación superior en América Latina y el Caribe: diez años después de la Conferencia Mundial de 1998. Cali: Pontificia Universidad Javeriana.

Tünnermann Bernheim, C. (2010). La declaración mundial sobre la educación superior en el siglo XXI: una lectura desde América Latina y el Caribe. Educación Superior y Sociedad, 10, 7-34.

Unión Europea (2015). Resolución del Parlamento Europeo, de 9 de septiembre de 2015, sobre las carreras profesionales de las mujeres en los ámbitos científico y académico y los techos de cristal existentes (2014/2251(INI)). Parlamento Europeo. no 60 (C 316 E) (22 de septiembre de 2017), disponible en: https://goo.gl/TtTNAF

Vasquez, E. G., y Lajud, C. A. (2016). Identidades y diversidades de género en la Escuela. Desafíos en pos de la igualdad. In C. V. Kaplan, (Ed.) (Ed.), Género es más que una palabra. Educar sin etiquetas (pp. 67-82). Buenos Aires: Miño y Dávila.

Veugelers, W., y de Groot, I. (2014). Educación Superior y desarrollo de la ciudadanía. In A. Teodoro y J. C. Beltrán (Eds.), Sumando voces. Ensayos sobre educación superior en términos de igualdad e inclusión social (pp. 45-52). Buenos Aires: Miño y Dávila.

Zugasti, N. (2014). La discriminación declarada por las propias personas afectadas. In ALTER. Grupo de investigación (Ed.), Los perfiles de la discriminación en España: Análisis de la Encuesta CIS3.000. Percepción de la discriminación en España (pp. 64-74). Madrid: Ministerio de Sanidad, Servicios Sociales e Igualdad. 